\title{
Is Broadcast Plus Multi-access Optimal for Gaussian Wireless Networks?
}

\author{
Amir F. Dana, Masoud Sharif, Radhika Gowaikar, Babak Hassibi and Michelle Effros \\ Department of Electrical Engineering \\ California Institute of Technology \\ Pasadena, CA 91125
}

\begin{abstract}
In this paper we show that "separation"-based approaches in wireless networks do not necessarily give good performance in terms of the capacity of the network. Therefore in optimal design of a wireless network, its total structure should be considered. In other words, achieving capacity on the subnetworks of a wireless network does not guarantee globally achieving capacity. We will illustrate this fact by considering some examples of multistage Gaussian wireless relay networks. We will consider a wireless Gaussian relay network with one stage in both fading and non-fading environment. We show that as the number of relay nodes, $n$, grows large, the capacity of this network scales like $\log n$. We then show that with the "separation"-based scheme, in which the network is viewed as the concatenation of a broadcast and a multi-access network, the achicvable rate scales as $\log \log n$ and as a constant for fading and non-fading environment, respectively, which is clearly sub-optimal.
\end{abstract}

\section{INTRODUCTION}

Although point-to-point information theory is well understood, little is known about the network information theory. The capacity region for many network scenarios is still unknown [1]. A type of max-flow min-cut theorem for general multiterminal networks is given in [1]. Using this theorem one can find different (not necessarily tight) upper bounds for achievable rates in arbitrary networks.

In wireline networks, for a family of problems known as multicast problems, it has been shown that the max-flow mincut upper bound can actually be achieved [2], [3], [4]. The goal in this problem is to convey information from one source to a number of destinations reliably. The network consists of a source, destinations and relay nodes. The links in the network represent memoryless independent channels with some known capacities.

This result is interesting in the following two aspects. First, the max-flow min-cut upper bound can be achieved in this problem, which is not the case for many networks. On the other hand, the achieviability proof of this capacity is based on a "separation"-based approach. This separation does not degrade the performance of the network. From the work of [2],[3], one can see that by using channel coding on each link, so as to make the links error-free, and by employing network

This work was supported in part by the National Science Foundation under grant no. CCR-0133818, by the office of Naval Research under grant no. N00014-02-1-0578, and by Caltech's Lee Center for Advanced Networking. coding on top and separated from the channel codes at the nodes, the max-flow min-cut upper bound is achievable.

In view of these results for wireline networks, one interesting and important question that arises is whether the same approach gives satisfactory results for multicast over wireless networks. We know that for the multicast problem over wireline networks making each link error-free does not degrade the performance of the network. Therefore one can decompose the network into smaller modules without degrading the performance of the network. The question is whether the same "separation"-based approach works for wireless networks.

In this paper, we will provide some examples of wireless networks for which this "separation"-based approach is not optimal and can cause severe degradation in the performance of the entire network. We will consider the simplest form of multicast problem with one source and one destination (unicast problem). This fact is illustrated by some examples of multi-stage Gaussian wireless relay networks. Therefore, for optimal operation of the network, its global structure should be considered and a coding scheme for the entire network, rather than for each individual link or sub-network is required. This fact is also noted in [5] for some different examples.

The paper is organized as follows. In the next section, we introduce our network model which is a multi-stage Gaussian wireless relay network. In Section 3 we will consider a single stage Gaussian wireless network. The performance of the "separation"-based approach for this network is analyzed for non-fading and fading cases. The maximum achievable rate is also compared to the simple scheme of "forward"ing at every relay node. In Section 4 we consider an example of a multistage Gaussian wireless relay network. For this network, we assume that each node can either "forward" or "decode and re-encode". Section 5 is further work and conclusion.

\section{NETWORK MODEL}

We consider multistage Gaussian wireless relay networks with one source of information denoted by $s$ and one destination node shown by $d$ (See Fig. 1). The network consists of $k$ stages of relay nodes to aid the communication between $s$ and $d$. We denote the $i$-th stage of relay nodes by $S_{i}\left(S_{0}\right.$ and $S_{k+1}$ denote nodes $s$ and $d$ respectively). The number of relay nodes in $S_{i}$ is denoted by $l_{i}$. Nodes in $S_{i}$ receive signal only from nodes in the previous stage $S_{i-1}$. We also 


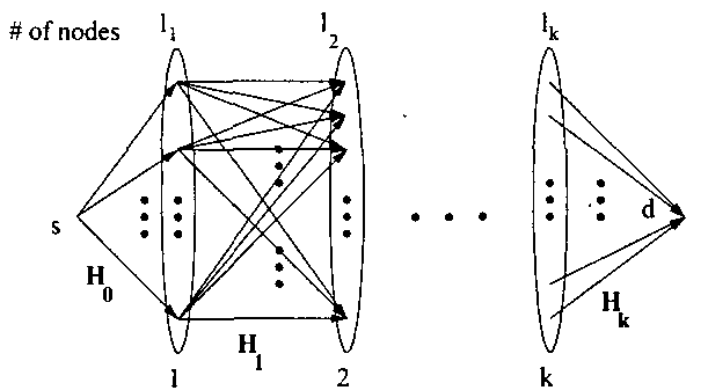

Fig. 1. A multi-stage Gaussian relay network

ignore the interference from other stages. We do not allow for any cooperation between the relay nodes. The channel between the nodes in stage $i$ and $i+1$ is assumed to be AWGN with channel gains denoted by $\mathbf{H}_{\mathrm{i}}$, which is a $l_{i} \times l_{i+1}$ matrix, i.e., $\mathbf{H}_{\mathbf{i}}[\mathbf{t}, \mathbf{m}]$ is the channel gain between node $t$ in stage $i$ and node $m$ in stage $i+1$. The noise introduced in every reception is assumed to be zero-mean unit variance complex Gaussian random variable with variance $\sigma_{n}^{2}$. The transmit power for all the nodes in $S_{i}$ is equal to $p_{i}$. In general, channels can be subject to fading. In that case, we assume local channel knowledge at the relay nodes and the source $s$ and global knowledge of the channel coefficients at destination $d$. Unless mentioned we assume that there is no fading present in the network. We assume synchronous reception at each node. $r_{i t}, t_{i t}$ and $\dot{v}_{i t}$ denote the received signal, the transmitted signal and the noise at node $t$ in stage $S_{i}$. Using the above notation, the network can be fully specified by Net $=\left(k,\left\{l_{i}\right\}_{i=1}^{k},\left\{p_{i}\right\}_{i=0}^{k},\left\{\mathbf{H}_{\mathbf{i}}\right\}_{i=0}^{k}, \sigma_{n}^{2}\right)$.

\section{EXAMPLE 1}

In this section we consider the class of networks introduced in the previous section with a single stage of relay nodes. We assume that the network has $n$ relay nodes. We consider both the cases when the network is subject to fading and when the channel gains between a relay node and the source and the destination are respectively equal to $g_{0}$ and $h_{0}$ for all the relay nodes.

We will study the maximum achievable rate for the network when a "separation"-based scheme is used. We will then mention a simple scheme that outperforms the "separation"based scheme.

\section{A. No fading}

In this case, using the notation of Section II, the network can be fully specified by

$\mathrm{Net}_{1}=\left(1,\{n\},\left\{p_{0}, p_{1}\right\},\left\{g_{0} *[11 \ldots 1], h_{0} *[11 \ldots 1]\right\}, \sigma_{n}^{2}\right)$.

l) "Separation"-based approach: In the "separation"based scheme, we decompose the network into two subnetworks: the broadcast network from the source, $s$, to the relay nodes and the multi-access network from the relay nodes to the destination, $d$. We view the network as the concatenation of these two sub-networks. We assume that the sub-networks are error-free. Given this we are interested in finding the maximum achievable rate with this scheme, $R_{s}$.

In this scheme because of the error-free operation of the sub-networks the maximum achievable rate, $R_{s}$, is actually $R_{s}=\min \left\{R_{B C}, R_{M A}\right\}$, where $R_{B C}$ and $R_{M A}$ are the maximum achievable sum-rate of the broadcast and the multiaccess network respectively.

The Gaussian broadcast network is a degraded broadcast channel for which the sum-capacity is given as [1]

$$
R_{B C}=\log \left(1+\frac{p_{0}\left|g_{0}\right|^{2}}{\sigma_{n}^{2}}\right)
$$

Also for the multi-access network from the relay nodes to the destination the capacity region is given in [1] and the sumcapacity is

$$
R_{M A}=\log \left(1+\frac{n p_{1}\left|h_{0}\right|^{2}}{\sigma_{n}^{2}}\right)
$$

Now we know the values for $R_{B C}$ and $R_{M A}$. By substituting these values in $R_{s}$ we have

$$
R_{s}=\log \left(1+\frac{\min \left(p_{0}\left|g_{0}\right|^{2}, n p_{1}\left|h_{0}\right|^{2}\right)}{\sigma_{n}^{2}}\right)
$$

2) "Forward": Another possible scheme for the above network is that the relay nodes normalize their received signal to power $p_{1}$ and "forward" it to the destination. Denoting the transmitted signal from $s$ by $t_{01}=x$, we have

$$
\begin{aligned}
r_{1 i} & =g_{0} x+v_{1 i} \\
t_{1 i} & =\sqrt{\frac{p_{1}}{\left|g_{0}\right|^{2} p_{0}+\sigma_{n}^{2}}}\left(g_{0} x+v_{1 i}\right) \\
r_{21} & =\frac{n h_{0} g_{0} \sqrt{p_{1}}}{\sqrt{\left|g_{0}\right|^{2} p_{0}+\sigma_{n}^{2}}} x+\frac{\sqrt{p_{1} h_{0}}}{\sqrt{\left|g_{0}\right|^{2} p_{0}+\sigma_{n}^{2}}} \sum_{i=1}^{n} v_{1 i}+v_{21}
\end{aligned}
$$

where as defined earlier, $r_{1 i}, t_{1 i}$ and $r_{21}$ are the received signal at relay node $i$, the transmitted signal from relay node $i$ and the received signal at the destination. From the above equation we see that signal to noise ratio at the destination is equal to

$$
S N R=\frac{. n^{2}\left|h_{0}\right|^{2}\left|g_{0}\right|^{2} p_{1} p_{0}}{\sigma_{n}^{2}\left(\sigma_{n}^{2}+p_{0}\left|g_{0}\right|^{2}+n p_{1}\left|h_{0}\right|^{2}\right)}
$$

therefore using the "forwarding" scheme the network can support a rate of

$$
R_{f}=\log \left(1+\frac{n^{2}\left|h_{0}\right|^{2}\left|g_{0}\right|^{2} p_{1} p_{0}}{\sigma_{n}^{2}\left(\sigma_{n}^{2}+p_{0}\left|g_{0}\right|^{2}+n p_{1}\left|h_{0}\right|^{2}\right)}\right)
$$

Comparing this with the rate achieved using "separation"based scheme we can find the condition for which "forward"ing is a better choice. In particular, for large enough $n$ we can see that $R_{f}=\Theta(\log n)$ while $R_{s}=\Theta(1)$. Therefore for large $n$, the. "Forward"ing scheme outperforms "separation"-based scheme. If we further impose $p_{0}=p_{1}$ then we have for $p_{0} \geq \frac{\sigma_{n}^{2}}{\{n-1) \max \left(n\left|h_{0}\right|^{2},\left|g_{0}\right|^{2}\right\}-\min \left\{n\left|h_{0}\right|^{2},\left\{\left.g_{0}\right|^{2}\right\}\right.}$ it is better to "forward" at relay nodes. 


\section{B. Fading}

In this section we consider a single stage relay network with $n$ relay node in the presence of fading. We assume that the channel gains are i.i.d zero-mean unit variance complex Gaussian random variables. This correspond to the Rayleigh fading scenario. Thus the network is specified as $\mathrm{Net}_{2}=$ $\left(1, n,\left\{p, \sigma_{r}^{2}\right\},\left\{\left[g_{1}, g_{2}, \ldots, g_{n}\right],\left[h_{1}, h_{2}, \ldots, h_{n}\right]\right\}, \sigma_{n}^{2}\right)$ where, for clarity, the connection from source to a relay node is shown by character $g$ and the connections from a relay node to destination is shown by character $h$.Similar to the previous section we compare the performance of the "separation"-based scheme with "forward"ing scheme.

1) "separation"-based scheme: Similar to the non-fading case, in the "separation"-based scheme we view the network as concatenation of a broadcast network (from source to relay nodes) and a multi-access network. We will design each of these sub-networks optimal in the sense of supportable rate.In this case the achievable rate, $R_{s}$, is again $\min \left\{R_{B C}, R_{M A}\right\}$ where $R_{B C}$ and $R_{M A}$ are the sum-capacities of a broadcast and a multi-access network. Note that, as mentioned in Section II, we assume the source and relay nodes have knowledge of their local channel gain and the destination has knowledge of all the channel gains in the network.

In the following paragraphs we first mention the results for the sum-capacity of the the broadcast and multi-access network in the presence of fading and then analyze the performance using the "forward"ing scheme.

- Broadcast network: For scalar Gaussian broadcast channels the ergodic sum-capacity, denoted in this paper by $R_{B C}$, is given by the following maximization problem[6]

$$
\begin{gathered}
R_{B C}=\max \mathrm{E} \log \left(1+\frac{1}{\sigma_{n}^{2}} \sum_{i=1}^{n} p_{i}\left|g_{i}\right|^{2}\right) \\
\text { subject to } \sum_{i=1}^{n} p_{i}=p \quad p_{i} \geq 0
\end{gathered}
$$

where as defined earlier, $g_{i}$ is the channel gain from the source to the $i$-th relay node. Furthermore the expectation is taken over the over the distribution of the channel gains. It can be verified that the optimal solution to the above maximization problem is to allocate all the power to the user with the strongest channel [6]. In this case, we have

$$
R_{B C}=\mathrm{E} \log \left(1+\frac{p}{\sigma_{n}^{2}}|g|^{2}\right)
$$

where $|g|^{2}=\max \left(\left|g_{1}\right|^{2}, \ldots,\left|g_{n}\right|^{2}\right)$. Now the cumulative density function (CDF) of $|g|^{2}$ is given as

$$
\operatorname{Pr}\left\{|g|^{2} \leq x\right\}=\left(1-e^{-x}\right)^{n}
$$

Therefore it can be shown that for large $n,|g|^{2}$ with high probability is or order $\log n$. More specifically, we have

$$
\operatorname{Pr}\left\{\ln \ln n \leq|g|^{2}-\ln n \leq \ln \ln n\right\}=1-O\left(\frac{1}{n}\right)
$$

Using this fact we have $R_{B C}=\Theta(\log (\log n))$.

- Multi-access network: The sum-capacity of the scalar
Gaussian nulti-access network with fading is derived in [7]. In this case the sum-capacity of the multi-access channel is given by

$$
R_{M A}=\mathrm{E} \log \left(1+\frac{\sigma_{r}^{2}}{\sigma_{n}^{2}} \sum_{i=1}^{n}\left|h_{i}\right|^{2}\right)
$$

Due to the law of large numbers as $n \rightarrow \infty$, we have $\frac{\sum_{i=1}^{n}\left|h_{i}\right|^{2}}{n} \rightarrow 1$. Therefore the sum-capacity of the $y_{m}$ Gaussian multi-access network with fading scales like $\log n$ for large $n$. We have stated this result as the following lemma.

We have summarized the above results in the following lemma.

Lemma 1. The capacities of the broadcast system between the source and the relay nodes and the multiaccess system between the relay nodes and the destination behave like $\log \log n$ and $\log n$, respectively, where $n$ is the number of relay nodes.

From the above discussion, we see that the maximum achievable rate in the network, when considered as a concatenation of a broadcast and multi-access network is of order $\log \log n$ as $n$ grows large, i.e. $R_{s}=\min \left\{R_{B C}, R_{M A}\right\}=$ $\Theta(\log \log n)$

2) "Forward": In this section we derive the scaling law for the capacity of the one stage relay network considered in this paper. We first mention an upper bound for the capacity of this network. Next, we show that we can obtain this scaling law by using "forward"ing scheme in the network. The main result of this section is given in the following theorem.

Theorem 1. Let $R_{\text {relay }}$ denote the capacity of the wireless relay network with $n$ relay nodes and the channel model as described in Section $111-B$. Then we have $R_{\text {relay }}=\Theta(\log n)$

Proof: The argument is similar to that of [8] and [9]. The upper bound is easily derived by allowing cooperation between the relay nodes. This scenario is clearly an upper bound for the capacity of the network with no cooperation. For this scenario the communication from the relay nodes to the receiver is like a $n$-antenna transmitter, 1 -antenna receiver system. In this case the upper bound is computed as

$$
\begin{aligned}
R_{\text {relay }} & \leq \mathrm{E} \log \left(1+\frac{1}{\sigma_{n}^{2}} \sigma_{r}^{2} \sum_{i=1}^{n}\left|h_{i}\right|^{2}\right) \\
& \leq \log \left(1+\frac{1}{\sigma_{n}^{2}} \sigma_{r}^{2} \sum_{i=1}^{n} \mathrm{E}\left|h_{i}\right|^{2}\right) \\
& \leq \log \left(1+n \frac{\sigma_{r}^{2}}{\sigma_{n}^{2}}\right)
\end{aligned}
$$

The lower bound is achieved by the "forwarding" scheme. In this scheme, the communication is done in two intervals. Using the notation of Section II, in the first interval the transmitter transmits a signal $t_{01}$. The relay node $i$ receives a noisy version of $t_{01}, r_{1 i}=g_{i} t_{01}+v_{1 i}$. In the second interval the relay nodes transmit a scaled version of what they have received. In this 
case, the transmitted signal from relay node $i$ is written as

$$
t_{1 i}=\sqrt{\frac{\sigma_{r}^{2}}{3 p+\sigma_{n}^{2}}} h_{i}^{*} g_{i}^{*} r_{1 i}=\sqrt{\frac{\sigma_{r}^{2}}{3 p+\sigma_{n}^{2}}} h_{i}^{*} g_{i}^{*}\left(g_{i} t_{01}+v_{1 i}\right)
$$

The scalar is chosen so that the average power of $t_{1 i}$ is $\sigma_{r}^{2}$ and also the signal parts add up coherently at the receiver. The received signal at the receiver can be written as

$$
\begin{aligned}
r_{21} & =\sum_{i=1}^{n} h_{i} t_{1 i}+v_{21} \\
& =\frac{\sigma_{r}\left(\left(\sum_{i=1}^{n}\left|h_{i}\right|^{2}\left|g_{i}\right|^{2}\right) t_{01}+\sum_{i=1}^{n}\left|h_{i}\right|^{2} g_{i}^{*} v_{1 i}\right)}{\sqrt{3 p+\sigma_{n}^{2}}}+v_{21}
\end{aligned}
$$

We can easily see that the average signal to noise power in the above equation is

$$
\mathrm{SNR}=\frac{n^{2} p \sigma_{r}^{2}}{\sigma_{n}^{2}\left(n \sigma_{r}^{2}+3 p+\sigma_{n}^{2}\right)}
$$

By using the central limit theorem we can show that as $n$ increases the maximum achievable rate using "forwarding" scheme, denoted by $R_{f}$, converges to $\frac{1}{2} \log (1+\mathrm{SN} R)$ where the factor of $\frac{1}{2}$ is because of the fact that the transmitter transmits half of the times. Now since $R_{f}$ is a lower bound for the capacity of wireless relay network, $R_{\text {relay }}$, we have

$$
R_{\text {relay }} \geq \frac{1}{2} \log \left(1+\frac{n^{2} p}{\sigma_{n}^{2}\left(n+3+\frac{\sigma_{n}^{2}}{p}\right)}\right)
$$

The upper bound and the lower bound suggest that $R_{\text {relay }}=$ $\Theta(\log n)$ and also that the "forwarding" scheme achieve this scaling for large $n$.

Based on the above discussion we have the following important observation. In this wireless setting for achieving the optimal rate of transmission we cannot view the Gaussian relay network with $n$ nodes as the concatenation of a broadcast system between the transmitter and the relay nodes and a multi-access system between the relay nodes and the receiver. In other words, if the network is designed so that the broadcast communication sub-network between the transmitter and the relay nodes perform optimally (in terms of rate) then the maximum rate that we can achieve is $\log \log n$ which is clearly less than the maximum achievable rate of the transmission in the main network.

\section{EXAMPLE 2}

The last network we consider in this paper is a multistage Gaussian relay network with $k$ stages and $n$ relay nodes in each stage. The transmit power at all the nodes is assumed to be $p$ and the noise variance is $\sigma_{n}^{2}$. We assume that all the channel gains are equal to some constant value $h$. Therefore the network is specified as Net $_{3}=$ $\left(k,\{n, \ldots, n\},\{p, \ldots, p\},\{[h, \ldots, h], \ldots,[h, \ldots, h]\}, \sigma_{n}^{2}\right)$.

In this network, we allow for two operations at each node: "forward" and "decode and re-encode". In the "decode and re-encode" scheme, the node, first decodes the message sent by the source successfully and then re-encode it by the same codebook used at the source and transmit it across the network. Decoding error-free at a node introduces a constraint on the maximum achievable rate of transmission from the source to the destination in the network. The goal is to find the optimal operation at each node so that the achievable rate is maximized. Note that because of the symmetry of the network, if in the optimal scenario, a relay node at stage $k$ "decodes and re-encodes" then without degrading the performance we can "decode and re-encode" at all of the other nodes in that stage. Therefore the problem is to find the stages at which to "decode and re-encode" is optimal. Let $D$ be the set of stages, excluding source and destination, at which the relay nodes "decode and re-encode". For instance, $D=\left\{S_{i}, S_{j}\right\}$ means relay nodes at stages $i$ and $j$ "Decode and re-encode" and $D=\emptyset$ means that all the relay nodes are "forward"ing. We refer to $D$ as a policy.

Now we are interested in the optimal policy, $D_{\text {opt }}$. Let $S N R_{i}(D)$ denote the signal to noise ratio at stage $i$ under policy $D$. Then it can be verified that the achievable rate under policy $D$, denoted by $R_{D}$ is given as

$$
\begin{aligned}
\quad R_{D}= & \min _{i \in \mathcal{I}} \log \left(1+S N R_{i}(D)\right) \\
\text { where } & \mathcal{I}=\left\{i \mid S_{i} \in D\right\} \cup\{k+1\}
\end{aligned}
$$

The above formula can be interpreted as follows. As mentioned earlier, by "decoding and re-encoding" at node $i \in D$ we introduce a constraint of $\log \left(1+S N R_{i}(D)\right)$ on the total rate of transmission from source to destination. Therefore $R_{D}$ is the minimum of these constraints.

As we see from (3), the important quantities that determine the performance of a policy, is the signal to noise ratio (SNR) at different stages. In the following paragraph we will state a lemma for computing these quantities

Lemma 2. Consider a multi-stage wireless relay network with $k$ stages specified as $\mathrm{Net}=$

$\left(k,\{n, \ldots, n\},\left\{p_{0}, p, \ldots, p\right\},\{[h, \ldots, h], \ldots,[h, \ldots, h]\}, \sigma_{n}^{2}\right)$

Therefore the transmit power for the source is $p_{0}$ and for all the relay nodes is $p$. Define $\rho_{0}=\frac{p_{0}}{\sigma_{n}^{2}}, \rho=\frac{p}{\sigma_{n}^{2}}$ and $c=|h|^{2}$. Then $S N R_{i}(\emptyset)$ (SNR when all the nodes are "forwarding") at the $i$-th stage is equal to

$$
\begin{aligned}
& S N R_{i}(\emptyset)=\frac{\rho_{0} \rho^{i-1} n^{2(i-1)} c^{i}}{X_{i}-\rho_{0} \rho^{i-1} n^{2(i-1)} c^{i}} \\
& X_{i}=\frac{1}{\alpha_{1}-\alpha_{2}}\left(\left(c \rho_{0}+1-\alpha_{2}\right) \alpha_{1}^{i}-\left(c \rho_{0}+1-\alpha_{1}\right) \alpha_{2}^{i}\right)
\end{aligned}
$$

where $\alpha_{1}$ and $\alpha_{2}$ are the (real) roots of the following equation

$$
z^{2}-\left(n^{2} c \rho+1\right) z+c \rho n(n-1)=0
$$

Using the above lemma, we can find the rate for the network considered in example 2 (i.e., $\mathrm{Net}_{3}$ ) under any policy $D=$ $\left\{S_{i_{1}}, S_{i_{2}}, \ldots, S_{i_{t}}\right\}$, where $i_{j+1}>i_{j}$ for all $j<t$. In order to find the rate, $R_{D}$, note that by "decoding and re-encoding" at some intermediate stage $S_{i_{j}}$, all the $n$ relay nodes in that stage 
act as a source with transmit power of $n^{2} p$ for next stages. Therefore the signal to noise ratio at stage $i_{j}$ is equal to the signal to noise ratio of the $i_{j}-i_{j-1}$-th stage of a network with transmit power of $p_{0}=n^{2} p$ at source and relay node power of $p$. Let $d_{j}=i_{j}-i_{j-1}$. Then using this observation and Lemma 2 , we can write the signal to noise at stage $i_{j}, j>1$ under policy $D=\left\{S_{i_{1}}, S_{i_{2}}, \ldots, S_{i_{t}}\right\}$ as

$$
\begin{aligned}
& S N R_{i_{j}}(D)=\frac{\rho^{d_{j}} n^{2 d_{j}} c^{d_{j}}}{X_{d_{j}}-\rho^{d_{j}} n^{2 d_{j}} c^{d_{j}}} \\
& X_{d_{j}}=\frac{1}{\alpha_{1}-\alpha_{2}}\left(\alpha_{1}^{d_{j}+1}-\alpha_{2}^{d_{j}+1}\right)
\end{aligned}
$$

where $\alpha_{1}$ and $\alpha_{2}$ are defined in (4). It can be verified from the above equation that $S N R_{i_{j}}$ is a decreasing function of $d_{j}$. Therefore if in the optimal policy there is one intermediate stage at which relay nodes should "decode and re-encode" at, then without loss of generality we can assume that relay nodes in next stages also "decode and re-encode". Thus the optimal policy can be written as

$$
D_{o p t}=\left\{S_{i_{0}}, S_{i_{0}+1}, \ldots, S_{k+1}\right\}
$$

and the corresponding rate $R_{o p t}$ can be written from (3) as

$$
R_{\text {opt }}=\log \left(1+S N R_{i_{0}}(\emptyset)\right)
$$

In other words, the rate is constrained by the SNR of the first "decode and re-encode" stage. Therefore, to find the optimal policy we need to find the stage with highest SNR when all the relay nodes are "forwarding". Theorem 2 summarizes the above discussion.

Theorem 2. Consider a multi-stage Gaussian wireless relay network with $k$ stages specified as

Net $=\left\{k,\{n, \ldots, n\},\{p, \ldots, p\},\{[h, \ldots, h], \ldots,[h, \ldots, h]\}, \sigma_{n}^{2}\right)$

Then the optimal policy for this network is given by $D_{\text {opt }}=$ $\left\{S_{i_{0}}, S_{i_{0}+1}, \ldots, S_{k+1}\right\}$ where $i_{0}$ is

$i_{0}=\left\{\begin{array}{cc}1 & \text { if } \rho \leq \frac{1}{|h|^{2}\left(n^{2}-n-1\right\}}, \\ 1+\min \left\{k,\left[\ln \frac{\left(|h|^{2} \rho+1-\alpha_{1}\right)\left(\alpha_{2}-|h|^{2} \rho n^{2}\right)}{\left(|h|^{2} \rho+1-\alpha_{2}\right)\left(\alpha_{1}-|h|^{2} \rho n^{2}\right)}\left(\ln \frac{\alpha_{1}}{\alpha_{2}}\right)^{-1}\right]\right\} \\ \text { otherwise. }\end{array}\right.$

$\lfloor x\rfloor$ denotes the floor of the real number $x$ and $\alpha_{1}$ and $\alpha_{2}<\alpha_{1}$ are the roots of the following equation

$$
z^{2}-\left(|h|^{2} n^{2} \rho+1\right) z+|h|^{2} \rho n(n-1)=0
$$

From the above theorem we have the following observations.

- In the low SNR regime, i.e. when $\rho$ is small enough, the optimal policy is to "decode and re-encode" at every stage. In this case the optimal rate is $R_{\text {opt }}=\log (1+$ $\left.|h|^{2} \rho\right)$. It can be shown that this property holds for any general multi-stage Gaussian relay network as defined in Section II where the transmit power at different stages are all decreasing functions of a single parameter.

- In the high SNR regime, i.e. when $\rho$ is large enough, the optimal policy is to "forward" at the relay nodes in the first stage and "decode and re-encode" at all the other stages, i.e., $D_{o p t}=\left\{S_{2}, S_{3}, \ldots, S_{k+1}\right\}$. Also the optimal rate, $R_{o p t}$ is given as

$$
R_{o p t}=\log \left(1+\frac{n^{2}|h|^{4} \rho^{2}}{(n+1)|h|^{2} \rho+1}\right) \quad \rho \text { large enough }
$$

In order to see this note that from (4), for large $\rho$ we have

$$
\begin{aligned}
& \alpha_{1}=\rho|h|^{2} n^{2}+\frac{1}{n}+O\left(\frac{1}{\rho}\right) \\
& \alpha_{2}=\frac{n-1}{n}+O\left(\frac{1}{\rho}\right)
\end{aligned}
$$

By plugging these values for $\alpha_{1}$ and $\alpha_{2}$ in (5), after some simplifications, $i_{0}$ can be written as

$$
i_{0}=1+\left\lfloor\frac{\ln \left(\rho|h|^{2} n^{3}\left(n^{2}-1\right)+O(1)\right)}{\ln \left(\rho|h|^{2} \frac{n^{3}}{n-1}+O(1)\right)}\right\rfloor
$$

From the above equation, we can see that as $\rho \rightarrow \infty$, the value inside $\left[\right.$. J goes to one and therefore $i_{0}=2$ for large enough $\rho$.

\section{CONCLUSION AND FUTURE WORKS}

In this paper we showed that the traditional approach of designing wired networks can not be always used in the wireless setting. By means of examples we have shown that designing the sub-networks of a wireless networks optimally (in terms of rate of transmission) does not necessarily imply good performance for the total network. For optimal operation of the network one should look at its global structure. Motivated by this, one interesting problem would be to develop sufficient conditions under which designing a wireless network locally guarantees good performance for the whole network. Another interesting problem is to find optimal designs in the limiting cases of high and low signal to noise ratio.

\section{REFERENCES}

[1] T. M. Cover and J. A. Thomas, Elements of information theory, Wiley, New York, 199].

[2] R. Alılswede, N. Cai, S.-Y, R, Li, and R. W. Yeung, "Network information flow," IEEE Trans. Info. Theory, vol. 46, no. 4, pp. 1204-1216, 2000.

[3] S.-Y. R. Li, R. W. Yeung, and N. Cai, "Linear network coding," IEEE Trans. Info. Theory, vol. 49, no. 2, pp. 371-381, 2003.

[4] Muriel Médard, "The effect upon chamnel capacity in wireless communications of perfect and imperfect knowledge of the channel," IEEE Trans. Info. Theory, vol. 46, no. 3, pp. 933-946, 2000.

[5] M. Effros, M. Médard, T. Ho, S. Ray, D. Karger, and R, Koetter, "Linear network codes: a unified framework for source channel, and network coding," invited paper to the DIMACS workshop on network info. theo., 2003 .

[6] D. N. C. Tse, "Optimal power allocation over parallel gaussian channels," Proc. Int. Symp. Information Theory, June 1997.

[7] S. Shamai and A. D. Wyner, "Information theoretic considerations for symmetric, cellular, multiple-access fading channels-part i," IEEE Trans. Inform. Thery, vol. 43, pp, 1877-1894, 1997.

[8] M. Gastpar and M. Vetterli, "On the asymptotic capacity of Gaussian relay networks," Proceeding of the 2002 International Symposium on Information Theory, p. 195, 2002.

[9] A. F. Dana and B. Hassibi, "On the power efliciency of sensory and ad-hoc networks," Submitted to the IEEE Transaction on information theory. 